\title{
EBP50 interacts with EGFR and regulates EGFR signaling to affect the prognosis of cervical cancer patients
}

\author{
ZHIQIANG PENG ${ }^{1}$, QIQI WANG ${ }^{1}$, YUE ZHANG ${ }^{1}$, JUNQI HE ${ }^{1,2}$ and JUNFANG ZHENG ${ }^{1,2}$ \\ ${ }^{1}$ Department of Biochemistry and Molecular Biology, Capital Medical University; \\ ${ }^{2}$ Beijing Key Laboratory for Tumor Invasion and Metastasis, Beijing International Cooperation Base \\ for Science and Technology on China-UK Cancer Research, Beijing 100069, P.R. China
}

Received May 18, 2016; Accepted July 22, 2016

DOI: 10.3892/ijo.2016.3655

\begin{abstract}
Ezrin-radixin-moesin-binding phosphoprotein-50 (EBP50) has a role in the occurrence and progression of multiple types of tumors. However, its role in cervical cancer (CC) remain unknown. EBP50 was reported to interact with epidermal growth factor receptor (EGFR) and regulate EGFR signaling in CC HeLa cells. In this study, the effect of EBP50 expression on CC cell proliferation and prognosis of CC patients by regulating EGFR signaling was investigated. We found that EBP50 expression level was significantly downregulated in CC tissues. EBP50 expression negatively correlated with $\mathrm{CC}$ cell proliferation, cell cycle and the activation of EGFR-mediated ERK signaling. EBP50 knockdown abolished its inhibition on EGF-induced ERK activation, suggesting EBP50 regulated EGFR signaling. In order to further explore EBP50 regulated EGFR signaling via interaction, we constructed EBP50_DD mutant which disrupted its interaction with EGFR. EBP50_DD overexpression attenuated the inhibition of EBP50_WT on EGFR-mediated ERK signaling, further revealing EBP50 regulated EGFR signaling via its interaction with EGFR. EGFR activation was associated with poor prognosis of CC patients. EBP50 could not predict the prognosis of all CC patients. However, after ruling out patients with $e g f r / E r b B$ mutation or copy number variation (CNV) and (chemo)radiation, which caused continuous EGFR activation and affected the prognosis of patients, respectively, EBP50 expression level exhibited the prognosis prediction
\end{abstract}

Correspondence to: Professor Junqi He or Dr Junfang Zheng, Department of Biochemistry and Molecular Biology, Capital Medical University, 10 Xitoutiao, You An Men, Beijing 100069 P.R. China

E-mail: zhengjf@ccmu.edu.cn

E-mail: jq_he@ccmu.edu.cn

Abbreviations: EBP50, ezrin-radixin-moesin-binding phosphoprotein-50; EGFR, epidermal growth factor receptor; CC, cervical cancer; PTEN, phosphatase and tensin homolog deleted on chromosome ten; PDGFR, platelet-derived growth factor receptor

Key words: EBP50, EGFR, cervical cancer ability, revealing EBP50 affected prognosis of CC patients via regulating EGFR signaling. In conclusion, EBP50 played an important role in CC cell proliferation and prognosis prediction of $\mathrm{CC}$ patients by interacting with EGFR and regulating EGFR signaling. EBP50 might be a potential precise therapeutic target or prognostic marker for $\mathrm{CC}$ patients.

\section{Introduction}

Cervical cancer (CC) is the fourth most common tumor type and leading cause of cancer death among women worldwide. Its incidence rate has increased in recent years $(1,2)$. Thus, to develop better prognostic and therapeutic strategies for CC, insight into the molecular and biologic mechanisms of tumorigenesis is critical.

Recent research suggests that Ezrin-radixin-moesinbinding phosphoprotein 50 (EBP50, also named NHERF1, NHERF), a scaffold protein containing two tandem PSD-95/ Discs Large/ZO-1 (PDZ) domains, plays an important role in tumor development and progression. EBP50 exerts tumor suppressing function in breast cancer, pancreatic cancer, biliary cancer, hepatocellular cancer, prostate cancer and glioblastoma (2-7). In addition, EBP50 was also reported to play the tumor-promoting roles. For example, EBP50 overexpression enhances cell invasion in breast cancer cells, and higher EBP50 level is associated with increasing tumor cytohistological grade, aggressive clinical behavior, and unfavorable prognosis in breast cancer (8-10). EBP50 could function as a positive regulator of Wnt signaling and might cause a malignant phenotype in hepatocellular carcinoma cells (11). EBP50 overexpression is involved in GBM invasiveness (12). However, the functional expression of EBP50 in CC cell proliferation and progression have not been reported.

Epidermal growth factor receptor (EGFR) was reported to correlate with cervical cancer (13). Overactivation of EGFR signaling is a hallmark of cancer and therapy strategy toward EGFR inhibition in cervical cancer has been ongoing (1). However, signaling proteins that connect the EGFR oncogenic cascade are poorly characterized (14). Scaffold proteins formed multiprotein complexes that were central to accurate coordination of signaling pathways (15). Abnormal expression of scaffold proteins has been linked to different types of cancer in human (16). EBP50 was reported to regulate EGFR 
signaling $(4,17,18)$. EBP50 overexpression induced a sustained activation of EGFR by increasing the level of EGFR at the HeLa cell surface (17). However, EBP50 is also reported to suppress EGFR activity by depleting the amount of EGFR at the cell surface (4). In addition, EBP50 blocked EGFR phosphorylation to inhibit EGF-induced breast cancer cell proliferation (18). Thus, the effect of EBP50 in regulating EGFR signaling was controversial. Especially, the regulatory effect of EBP50 on EGFR signaling in CC patients remains unclear.

In this study, we first found low expression of EBP50 in CC samples and EBP50 expression level negatively correlated with CC cell proliferation, cell cycle and EGFR signaling activation. EBP50 knockdown abolished the inhibition on EGF-induced ERK signaling activation. In order to verify EBP50 regulated EGFR signaling via interaction, we constructed EBP50 mutant DD (S279D/S301D) which disrupted the interaction with EGFR. The overexpression of DD mutant attenuated its inhibition on EGFR-mediated signaling, revealing EBP50 regulated EGFR signaling via interaction with EGFR. Further evidence showed that EBP50 could predict the prognosis of $\mathrm{CC}$ patients after ruling out the patients with mutation/copy number alteration of egfr/ $E r b B$ gene and (chemo)radiation, which led to continuous activation of egfr gene affecting patient prognosis, respectively. EBP50 could be the precise therapeutic target and prognostic marker for $\mathrm{CC}$ patients.

\section{Materials and methods}

Tissue microarray data. The IHC-based protein expression data including high-resolution images were viewed and downloaded from the Human Protein Atlas web portal (www. proteinatlas.org).

The Cancer Genome Atlas (TCGA) data. The TCGA data about mRNA (RNA Seq v2) and protein (RPPA) expression levels in cervical cancer patients were obtained from https:// www.synapse.org/. The EBP50 mRNA level and c-Raf_pS338 protein level were used in this study. Clinical data was downloaded from cBioPortal database (www.cbioportal.org).

Gene set enrichment analysis. The association between phenotypes, biological processes/pathway and EBP50 expression level was analyzed using gene set enrichment analysis (GSEA v2.2, http://www.broad.mit.edu/gsea/). GSEA calculates a gene set enrichment score (ES) that estimates whether genes from pre-defined gene set [obtained from the Molecular Signatures Database, MSigDB, http://software.broadinstitute. org; genesets:REGULATION_OF_CELL_PROLIFERATION, POSITIVE_REGULATION_OF_CELL_CYCLE (annotated by the GO term GO: 0042127 and GO: 0045787, respectively), EGFR_UP.V1_DN, REACTOME_PERK_REGULATED_ GENE_EXPRESSION, EGFR_UP.V1_UP] are enriched among the highest- (or lowest-) ranked genes or distributed randomly. Default settings were used. Thresholds for significance were determined by permutation analysis $(1,000$ permutations). False discovery rate (FDR) was calculated. A gene set is considered significantly enriched when the FDR score is $<0.05$.
Plasmids. shEBP50 constructs (pSuper.puro shEBP50) were kind gifts of Dr M.J. Wheelock (University of Nebraska Medical Center, Omaha, NE, USA).

GST-tagged EBP50_WT and GST-tagged EBP50 mutant (S279D/S301D, DD) plasmids, pBK-CMV-Hemagglutinin (HA)-EBP50_WT and pBK-CMV-HA-EBP50 mutant (DD) expression plasmids were kindly provided by Dr Randy Hall from Emory University.

Cell culture, transfection and cell treatments. The human cervical carcinoma cell line HeLa was obtained from the American Type Culture Collection (ATCC, Manassas, VA, USA). HeLa was grown in Dulbecco's modified Eagle's medium (DMEM, Gibco) (19) at $37^{\circ} \mathrm{C}$ and $5 \% \mathrm{CO}_{2}$. The media were supplemented with $10 \%$ fetal bovine serum (FBS, Hyclone, Logan, UT, USA) and 1\% antibiotic-antimycotic agent (Life Technologies, Inc., Grand Island, NY, USA). Cells were grown to $80 \%$ confluency for use. Transfections were performed by Lipofectamine 2000 (Invitrogen, CA, USA) with plasmid DNA following the protocol as reported before (3).

HeLa cells were serum starved overnight, then treated with 100 ng/ml EGF (Sigma-Aldrich Chemical Corp., St. Louis, MO, USA) for $5 \mathrm{~min}$ at $37^{\circ} \mathrm{C}$ to detect the effect of EBP50 knockdown, EBP50_WT or EBP50_DD overexpression on EGFR-mediated signal transduction pathways.

Stable transfection. For stable knockdown, shEBP50 constructs were transfected into HeLa cells following the protocol. Two days following transfection, cells were transferred to $90-\mathrm{mm}$ plates and cultured in selection medium with $0.5 \mu \mathrm{g} / \mathrm{ml}$ puromycin (Sigma-Aldrich) to obtain EBP50 knockdown cells. Resistant colonies formed were harvested and cultured for at least a month, then the fractions were used for analysis of EBP50 expression by western blotting, with GAPDH expression as a protein loading control. Stably-transfected cells were maintained and passaged in culture medium with puromycin $(0.25 \mu \mathrm{g} / \mathrm{ml})(18)$. HeLa cells stably knocked down with the shEBP50 plasmids were called HeLa_shEBP50.

Western blotting. Samples were run on $10 \%$ sodium dodecyl sulfate (SDS)-polyacrylamide gels (PAGE) and transferred to PVDF membranes. The blots were blocked in blocking buffer (5\% non-fat dry milk in TBST buffer) for $1 \mathrm{~h}$ at room temperature and then incubated with primary antibodies $(1: 1,000)$ in blocking buffer overnight at $4^{\circ} \mathrm{C}$. The blots were washed three times with TBST buffer and incubated for $1 \mathrm{~h}$ at room temperature with a horseradish peroxidase (HRP)-conjugated anti-mouse IgG or anti-rabbit IgG secondary antibody (1:3000; Amersham Biosciences, Piscataway, NJ, USA) in blocking buffer. Finally, the blots were washed three times with TBST buffer and visualized via enzyme-linked chemiluminescence using the electrochemiluminescence (ECL) kit (Applygen Technologies Inc., Beijing, China) (3). The levels of immunoreactivity were semi-quantitatively analyzed by NIH Image 1.62 software.

The primary antibody specific for EBP50 was purchased from BD Biosciences (San Jose, CA, USA), HA was from MBL (Nagoya, Japan). Other primary antibodies specific for GAPDH, phospho-EGFR (Tyr1173), phospho-ERK1/2 
A

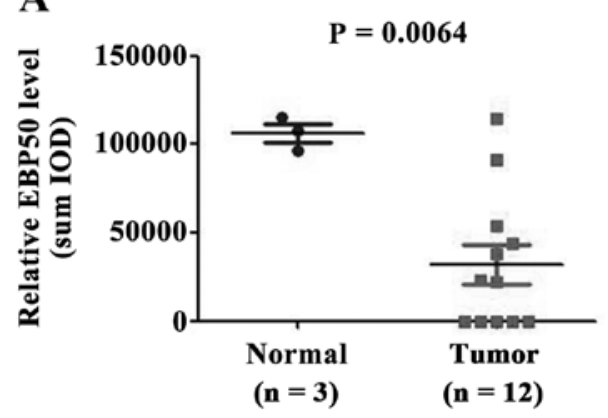

B

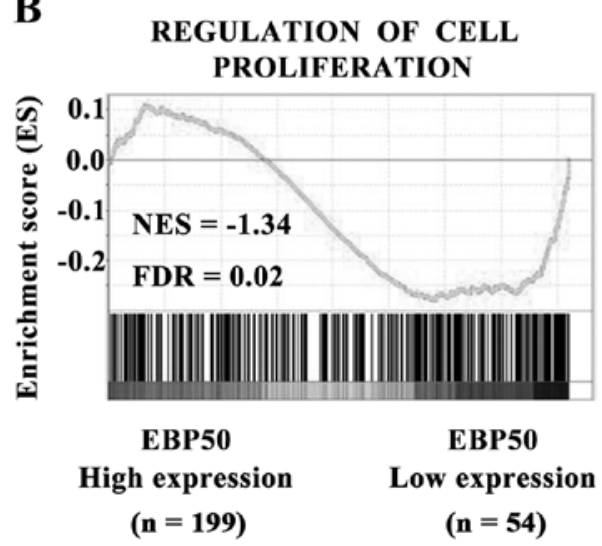

\begin{tabular}{|c|c|c|}
\hline EBP50 Level & Normal & Tumor \\
\hline High & $\mathbf{0}(\mathbf{0} \%)$ & $\mathbf{0}(\mathbf{0} \%)$ \\
\hline Medium & $\mathbf{1 0 0 ( 1 0 0 \% )}$ & $\mathbf{1}(\mathbf{8 . 3} \%)$ \\
\hline Low & $\mathbf{0 ( 0 \% )}$ & $\mathbf{6 ( 5 0 \% )}$ \\
\hline Not detected & $\mathbf{0 ( 0 \% )}$ & $\mathbf{5}(\mathbf{4 1 . 7} \%)$ \\
\hline
\end{tabular}

C

POSITIVE REGULATION OF CELL CYCLE

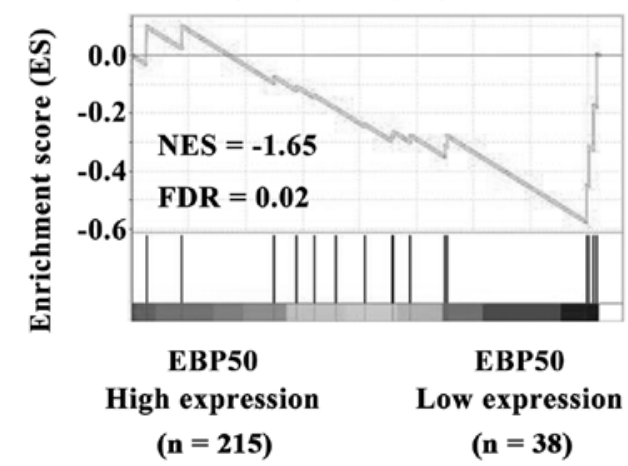

Figure 1. EBP50 expression level negatively correlates with cell proliferation and cell cycle in cervical cancer (CC) patients. (A) Downregulation of EBP50 expression level in CC samples. Left panel, scatter plot for EBP50 expression level in CC ( $\mathrm{n}=12$ ) and normal ( $\mathrm{n}=3$ ) tissues (the Human Protein Atlas). Significance between the 2 populations was determined with a two tailed t-test. Right panel, the level and percentage of EBP50 protein in CC patients and normal tissues. (B) Enrichment plots of gene expression signatures for cell proliferation and cell cycle according to EBP50 mRNA expression levels by gene set enrichment analysis (GSEA) of TCGA dataset. Samples were divided into high and low EBP50 expression groups. False discovery rate (FDR) gives the estimated probability that a gene set with a given normalized ES (NES) represents a false-positive finding; FDR $<0.05$ is a widely accepted cutoff for the identification of biologically significant gene sets.

(Thr202/Tyr204), total EGFR and ERK1/2 were all bought from Cell Signaling Technology (Beverly, MA, USA).

GST pull-down assay. Glutathione S-transferase (GST) fusion proteins were purified from bacteria using glutathioneSepharose 4B beads (Sigma-Aldrich) according to the manufacturer's protocol. The GST pull-down assay was performed as described previously (20). Briefly, equal amounts of GST or GST-EBP50_WT, GST-EBP50_DD fusion protein beads, were incubated with equal amounts of cell lysates. After incubation at $4^{\circ} \mathrm{C}$ for $2 \mathrm{~h}$, the beads were washed with ice-cold wash buffer (100 mM NaCl, $10 \mathrm{mM}$ HEPES, pH 7.4, $5 \mathrm{mM}$ EDTA, $1 \mathrm{mM}$ benzamidine, 3\% BSA and 0.1\% Tween-20). Proteins were then eluted with SDS sample buffer $(50 \mathrm{mM}$ Tris/HCl, $100 \mathrm{mM}$ DTT, $2 \%$ SDS, $0.1 \%$ bromophenol blue and $10 \%$ glycerol), and detected with western blotting.

Co-immunoprecipitation assay. Co-immunoprecipitation was performed as described before (21). Briefly, COS-7 cells transiently transfected with HA-EBP50_WT or HA-EBP50_DD were harvested and then lysed in $1 \mathrm{ml}$ of ice-cold lysis buffer (10 mM HEPES, $50 \mathrm{mM} \mathrm{NaCl}, 5 \mathrm{mM}$ EDTA, $1 \mathrm{mM}$ benzamidine, $0.5 \%$ Triton $\mathrm{X}-100, \mathrm{pH} 7.4$ ). Lysates were solubilized, clarified and then incubated with anti-HA or anti-EGFR antibody, prebounded with protein A\&G beads (Calbiochem, CA, USA). After washing with an ice-cold lysis buffer three times, the immunoprecipitated proteins were eluted from the beads with SDS sample loading buffer. The eluted samples were then analyzed by western blotting.

Statistical analyses. Statistical analyses were performed using the SPSS 18.0 (SPSS Inc, Chicago, IL, USA) and Graphpad Prism 5 (Graphpad software Inc, San Diego, CA, USA). Group distributions were compared using the Student's t-test. A value of $\mathrm{P}<0.05$ was considered statistically significant. The association between the EBP50 expression level and patient's overall survival (OS) was assessed by Kaplan-Meier method.

\section{Results}

EBP50 expression negatively correlates with cell proliferation and cell cycle in cervical cancer $(C C)$ patients. To investigate the role of EBP50 in CC, the expression level of EBP50 in CC tissues was first analyzed. Immunohistochemistry data from tissue microarrays of the Human Protein Atlas dataset were obtained to analyze the expression level of EBP50 between cervical cancer and normal cervix tissues. EBP50 protein expression level was significantly downregulated in CC tissues compared with the normal controls ( $\mathrm{P}<0.01$, Fig. $1 \mathrm{~A})$. In $41.7 \%$ of tumor tissues EBP50 was not detected. $50 \%$ of tumor tissues weakly expressed EBP50, and $8.3 \%$ of tumor tissues expressed EBP50 in medium level (Fig. 1A). In contrast with 
A

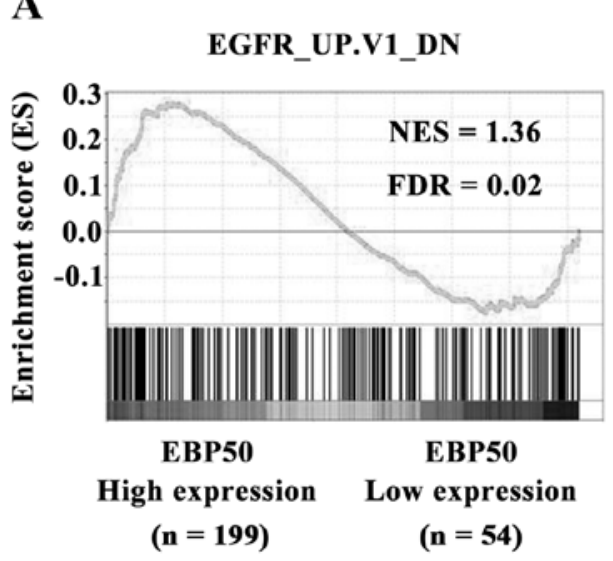

C

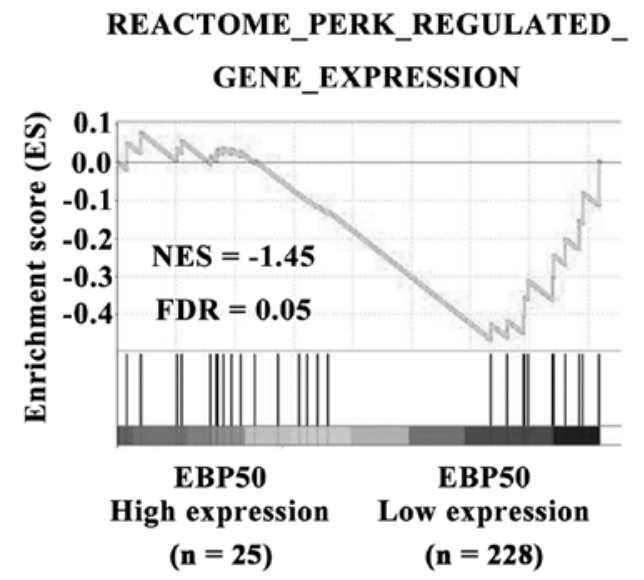

B

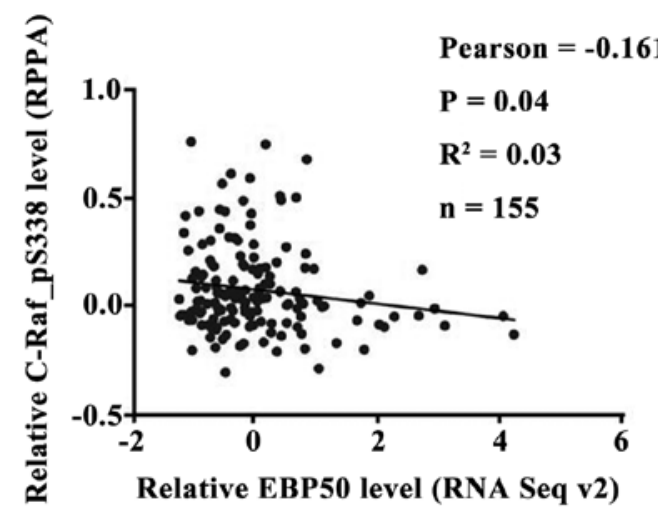

D
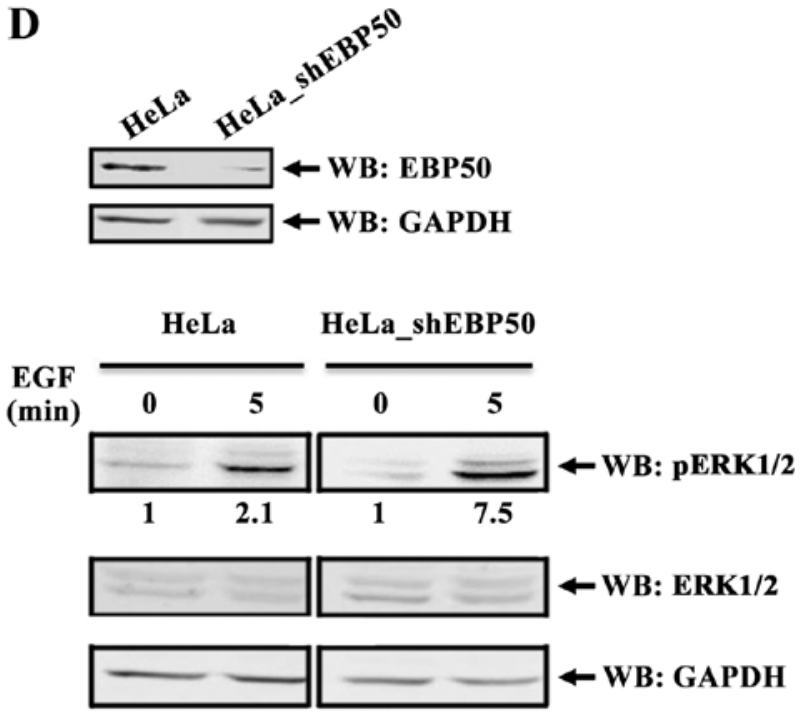

Figure 2. EBP50 expression is negatively associated with EGFR signaling and EBP50 knockdown abolishes its inhibition on EGF-induced ERK signaling. (A) EBP50 expression is negatively associated with EGFR activation. GSEA of TCGA data depicting the enrichment of EGFR pathway activation gene set Samples were divided into high and low EBP50 expression groups. EGFR gene set was enriched in EBP50 high expression group. (B) EBP50 expression level is negatively associated with Raf activation level. The negative association between c-RAF_pS338 protein level and EBP50 mRNA level in 155 cervical cancer patients from TCGA dataset. (C) EBP50 expression is negatively associated with ERK activation. GSEA of TCGA data depicting the enrichment of ERK activation gene set. Samples were divided into high and low EBP50 expression groups. (D) EBP50 knockdown abolished its inhibition on EGF-stimulated ERK1/2 phosphorylation in HeLa cells. ERK1/2 activation level in shEBP50 cells was significantly higher compared with that in its control cells upon EGF treatment. The effect of EBP50 on phosphorylation level of ERK was analyzed by western blotting.

tumor tissues, normal cervical tissues expressed EBP50 in medium level at $100 \%$. Taken together, EBP50 was expressed in medium level for all normal cervix tissues, whereas weak or no expression was detected in large part of $\mathrm{CC}$ tissues despite medium level of EBP50 in CC tissue of one case. EBP50 expression level was significantly downregulated in CC tissues.

Low expression level of EBP50 in CC tissues indicated that EBP50 played a role in CC tumorigenesis, so we further studied the biological effect of EBP50 in CC samples. The CC patients in the TCGA dataset was divided into high EBP50 expression and low EBP50 expression groups, and the correlations between cell proliferation/cell cycle and EBP50 expression were further analyzed using the GSEA method. As shown in Fig. 1B, the gene set of cell proliferation and cell cycle, was highly enriched in EBP50 low expression group (Fig. 1C, FDR <0.05). These data suggested a negative correlation between EBP50 expression level and cell proliferation or cell cycle in clinical CC samples. Low expression level of EBP50 in CC tissues contributed to $\mathrm{CC}$ cell proliferation or cell cycle.

EBP50 expression is negatively associated with EGFR signaling activation in cervical cancer. To investigate the mechanism by which EBP50 suppressed CC cell proliferation and cell cycle, we studied the regulatory effect of EBP50 on EGFR signaling since EGFR is an important growth promoting factor in $\mathrm{CC}(22,23)$ and EBP50 was reported to regulate EGFR signaling via its interaction with EGFR $(4,17)$. We performed GSEA to identify the correlation between EBP50 expression level and EGFR signaling. EGFR gene set (EGFR_UP.V1_DN, the downregulated gene set after EFGR pathway was activated, indicating the activation of EGFR pathway) was highly enriched in the EBP50 high expression group (Fig. 2A, FDR <0.05), revealing that EBP50 negatively correlated with EGFR signaling activa- 
A

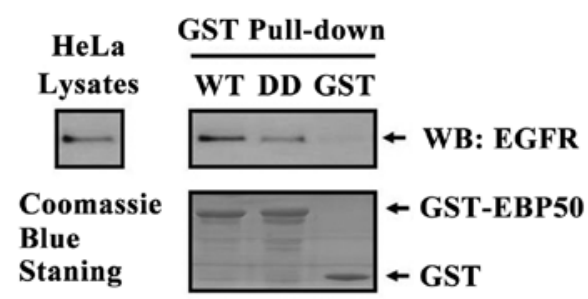

B

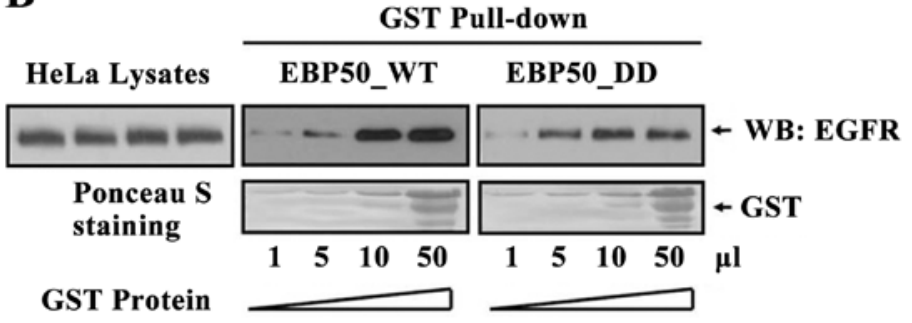

C

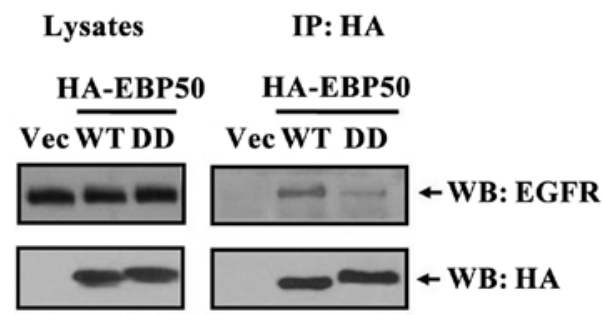

Figure 3. EBP50_DD mutation disrupts its interaction with EGFR. (A) GST pull-down result showed that EBP50_DD (S279D/S301D-DD) mutation disrupted the interaction between EGFR and EBP50. Lysates from HeLa cells expressing EGFR were utilized in pull-down experiments with beads loaded with GST or GST-EBP50_WT or GST-EBP50_DD. The precipitates were run on 12\% SDS-PAGE gels, blotted and visualized with an anti-EGFR antibody. Coomassie blue staining revealed equal loading of the fusion proteins. Less EGFR was pulled down by GST-EBP50_DD fusion protein than GST-EBP50_WT, proving that EBP50_DD mutation disrupted the interaction between EGFR and EBP50. (B) Differential increase of EGFR pulled down by different dose of GSTprotein further verified EBP50_DD mutation disrupted the interaction between EGFR and EBP50. Lysates from HeLa cells expressing EGFR were utilized in pull-down experiments with beads loaded with four different concentrations of GST-EBP50_WT or GST-EBP50_DD from 1 to 50 $\mu 1$. The precipitates were run on $12 \%$ SDS-PAGE gels, blotted and visualized with an anti-EGFR antibody. Ponceau S staining revealed the equal loading of proteins in the same concentration of the GST fusion proteins. (C) Co-immunoprecipitation results also revealed that EBP50_DD mutation disrupted the interaction between EGFR and EBP50. COS-7 cells were transfected with pBK-CMV-HA vector or pBK-CMV-HA-EBP50_WT plasmid or pBK-CMV-HA-EBP50_DD plasmid. The total lysates were blotted with anti-HA or anti-EGFR antibody to visualize the EBP50 and EGFR expression (left panel). All lysates were solubilized and incubated with anti-HA antibody coupled to beads in order to immunoprecipitate the HA-EBP50. The immunoprecipitates were probed with an anti-EGFR antibody (right panel).

tion. To further investigate the effect of EBP50 expression on EGFR-mediated signal activation, the regulatory effect of EBP50 on c-Raf and ERK, which are the downstream molecules for EGFR-mediated signaling, were further analyzed. TCGA dataset was used to analyze the correlation between EBP50 and c-Raf_pS338 expression levels. EBP50 expression level is negatively correlated with c-Raf_pS338 expression levels (Fig. 2B). ERK activation gene set was enriched in the low EBP50 expression group (Fig. 2C, FDR <0.05), further suggesting a negative correlation between EBP50 expression level and EGFR/ERK signaling in clinical CC samples.

To verify the regulatory effect of EBP50 on EGFR-mediated ERK signaling, we further knocked down the expression of EBP50 in HeLa cells to detect EGF-induced ERK phosphorylation level. As shown in Fig. 2D, EBP50 knockdown relieved its inhibition on EGF-induced ERK phosphorylation level in HeLa cells. After 5 min of EGF stimulation, ERK phosphorylation level in HeLa cells increased 2.1-fold over basal level, whereas ERK phosphorylation level in EBP50 knockdown HeLa cells increased 7.5-fold over basal level. These results confirmed that EBP50 inhibited EGF-induced ERK phosphorylation in $\mathrm{CC}$ cells.

EBP50 mutant DD disrupts its interaction with EGFR. EBP50 regulated multiple signaling pathways, such as EGFR, PDGFR, PTEN and Wnt signaling pathways (11,17,24-28). Thus, that EBP50 knockdown regulated EGF-induced ERK signaling can not exclude other signaling pathway-mediated ERK signaling. To verify EBP50 regulated EGFR signaling via its interaction with EGFR in CC tissues, we constructed EBP50 mutant DD (29) and detected their interaction by GST pull-down assay. As shown in Fig. 3A, the amount of endogenous EGFR in HeLa cells pulled down by GST-EBP50_DD was much less than that by GST-EBP50_WT, suggesting that EBP50_DD mutation retarded the association of EGFR and EBP50. 


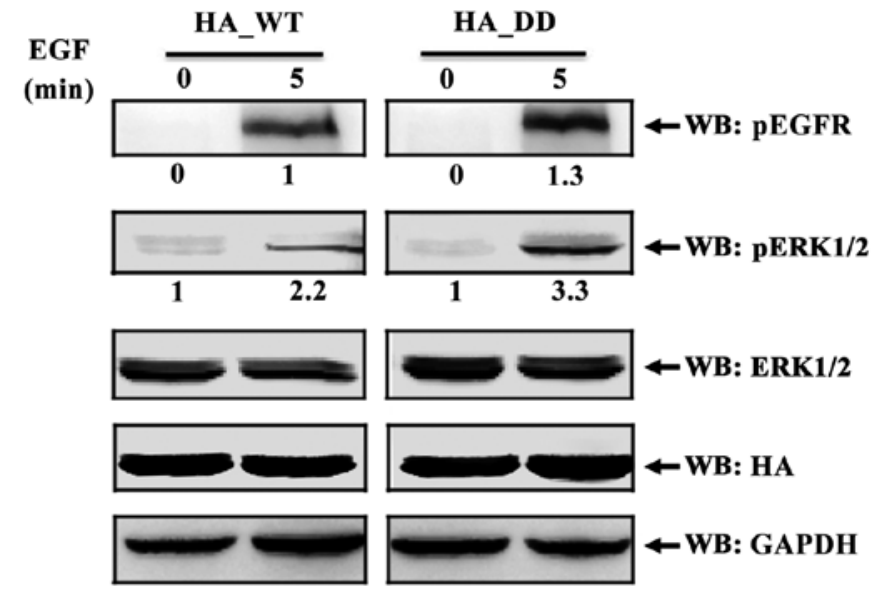

Figure 4. EBP50 inhibits EGF-induced EGFR and ERK phosphorylation via interacting with EGFR. EBP50_DD overexpression attenuated its inhibition on EGFR signaling. HeLa cells overexpressing EBP50_WT or EBP50_DD were seeded on 35-mm wells (6-well culture dish) and were treated with serum-free medium overnight. Serum-starved HeLa cells were stimulated for $5 \mathrm{~min}$ respectively with $100 \mathrm{ng} / \mathrm{ml} \mathrm{EGF}$ at $37^{\circ} \mathrm{C}$. The cells were solubilized in 1X SDS-PAGE sample buffer. Phosphorylation levels of EGFR and ERK in the whole cell lysates were detected by western blot analysis using an antiphospho-EGFR and phospho-ERK1/2 antibodies. Their activation levels were quantified after normalization by EGFR and ERK. The data presented are representative of a minimum of three independent experiments. EBP50_DD attenuated its inhibition on EGFR signaling.

To further test whether EBP50_DD mutation retarded the association of EGFR and EBP50, we then used different dose of GST-EBP50_WT and GST-EBP50_DD (1-50 $\mu \mathrm{l})$ fusion protein to pull down endogenous EGFR from the same amount of HeLa cell lysates, respectively. With the increase of both GST-EBP50_WT and GST-EBP50_DD fusion protein amount, the amount of EGFR pulled down increased correspondingly. However, the amount of EGFR pulled down by GST-EBP50_DD was less than that by GST-EBP50_WT in each dose (Fig. 3B), further revealing EBP50_DD mutation retarded the association of EGFR and EBP50.

To verify the results, we used co-immunoprecipitation assay to further investigate the disruption effect of EBP50_DD mutation on its interaction with EGFR in cellular context. Results showed that less EGFR co-immunoprecipitated with EBP50_DD than EBP50_WT (Fig. 3C), which was consistent with GST pull-down results, and again demonstrated that EBP50_DD mutation disrupted the interaction of EGFR with EBP50.

EBP50 regulates EGFR/ERK signaling via its interaction with EGFR. To confirm the inhibitory effect of EBP50 on EGFR/ERK signaling by interacting with EGFR in CC cells, we transiently transfected HA-EBP50_WT and HA-EBP50_ DD plasmids into HeLa cells, respectively. As shown in Fig. 4, EBP50_DD attenuated the inhibition of EBP50 on EGFR-mediated ERK phosphorylation in HeLa cells. After 5 min of EGF stimulation, EGFR phosphorylation level was increased 1.3-fold over basal level and ERK phosphorylation level was increased 3.3-fold over basal level in HA-EBP50_DD transfected HeLa cells, whereas EGFR phosphorylation level was increased 1-fold over basal level and ERK phosphorylation level was increased 2.2-fold over basal level in HA-EBP50_
A
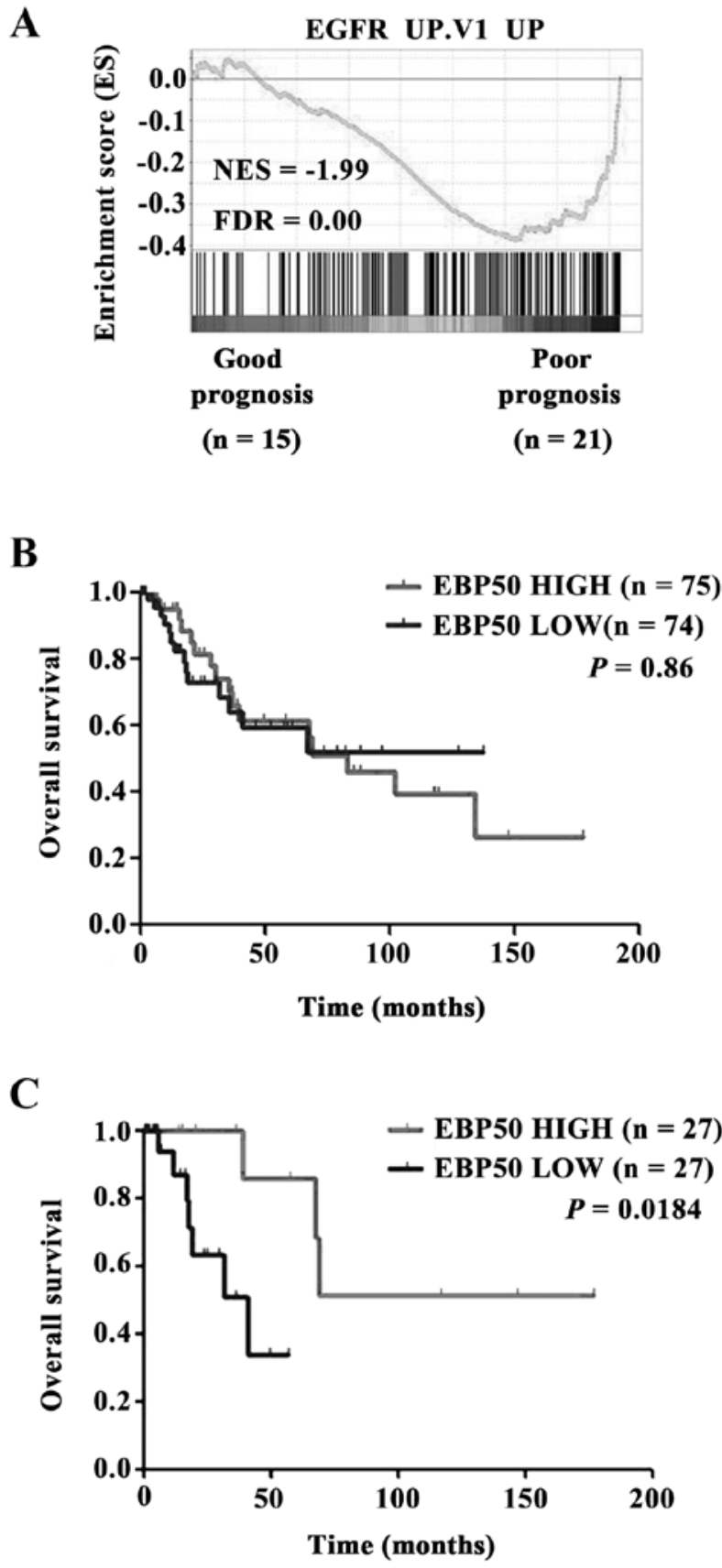

Figure 5. EBP50 expression correlates with poor prognosis of CC patients without $e g f r / E r b B$ alteration and (chemo)radiation. (A) Enrichment plots of EGFR activation gene set according to EBP50 mRNA expression levels by GSEA of TCGA dataset. Samples were divided into good (5 years, living) and poor (3 years, deceased) prognosis groups. (B) Kaplan-Meier (KM) analysis of OS based on EBP50 expressions levels in $149 \mathrm{CC}$ patients from TCGA dataset. The median level of EBP50 was used as the cutoff. (C) KM analysis of OS based on EBP50 expressions levels in 54 cases without mutation or CNA of $e g f r / E r b B$ gene and chemoradiotherapy from TCGA dataset. The median level of EBP50 was used as the cutoff.

WT transfected HeLa cells, respectively. The activation levels of EGFR and ERK in HA-EBP50_DD transfected HeLa cells were higher than those in HA-EBP50_WT transfected HeLa cells. The result indicated that disrupted interaction of EBP50 with EGFR led to decreased inhibition on EGFR signaling. This result further verified EBP50 inhibited EGFR-mediated ERK phosphorylation via interaction with EGFR in CC cells. 
Low EBP50 expression level is correlated with poor prognosis in cervical cancer. Activation of EGFR was associated with poor prognosis in CC (30). The analysis of TCGA dataset revealed EGFR pathway was over-activated in CC patients with poor prognosis (3 years, dead) compared with those with good prognosis (5 years, living) (Fig. 5A). The clinical prognosis relevance of EBP50 expression level with CC patients was also evaluated. Unexpectedly, EBP50 expression level in TCGA dataset had no significant prognosis predictive ability for all $\mathrm{CC}$ patients (Fig. 5B). Considering that EGFR/ $E r b B$ gene mutation or copy number alteration (CNA) will lead to continuous activation of EGFR signaling and (chemo) radiation will influence the prognosis of CC patients. When $C C$ patients with $E G F R / E r b B$ gene mutation or copy number alteration (CNA) and (chemo)radiation were omitted, KaplanMeier survival analysis showed that high EBP50 expression group had better outcomes than low EBP50 expression group in terms of survival duration (Fig. $5 \mathrm{C}, \mathrm{P}<0.05$ ). These results further indicated that EBP50 affected prognosis of CC patients via regulating EGFR signaling pathway.

\section{Discussion}

EBP50 is an adaptor protein consisting of two PDZ domains and one ezrin-binding region. Through these functional domains, EBP50 interacts with many proteins and regulates their functions $(11,17,24,25,28,31)$. For example, EBP50 can enhance the stability of phosphatase and tensin homologue deleted on chromosome 10 (PTEN) protein and recruit PTEN to cell membrane in breast cancer cells $(24,31)$, stabilize $\beta$-catenin at cell membrane of mouse embryonic fibroblast (MEF) cells $(11,28)$, promote platelet-derived growth factor receptor (PDGFR) phosphorylation in normal cells $(25,28)$. In this study, we found that EBP50 expression negatively correlated with EGFR-mediated ERK activation. EBP50 knockdown abolished its inhibition on EGF-induced ERK activation. PTEN and PDGFR, can also regulate ERK signaling (32-34). EBP50 could regulate EGFR-mediated ERK signaling via direct interaction with EGFR (17) or indirectly recruiting PTEN to EGFR (35). To elucidate whether EBP50 modulate ERK signaling via interaction with EGFR, we constructed EBP50 mutant DD which destroyed the interaction with EGFR. The overexpression of EBP50 mutant DD attenuated the inhibition of EBP50 on EGF-induced EGFR and ERK activation in CC cells, further verifying EBP50 regulated EGFR signaling via direct interaction with EGFR in CC.

We speculated the following mechanisms by which scaffold protein EBP50 regulated EGFR signaling via direct interaction. Scaffold proteins were shown to promote conformational changes of their binding partners $(36,37)$. It was possible that when EBP50 bound with EGFR, the conformation of EGFR changed and EGFR was not easily phosphorylated. EBP50 altered the subcellular localization or membrane localization of EGFR in biliary carcinoma cells (4). In CC cells, EBP50 might also alter the subcellular localization or membrane localization of EGFR to regulate EGFR-mediated signaling. These aspects need to be further investigated.

Previously we reported that EBP50 overexpression in HeLa cells could suppress HeLa cell proliferation and anchorage-independent growth (3). In this study, we found that
EBP50 was significantly downregulated in CC tissues. EBP50 expression negatively correlated with $\mathrm{CC}$ cell proliferation and cell cycle by interacting with EGFR and suppressing EGFR signaling. We further found EBP50 could predict prognosis of CC patients without continuous activation mutation/copy number alteration of egfr/ErbB gene and (chemo)radiation which affected patient prognosis. These data supported a novel tumor suppressor role of EBP50 in cervical cancer and new mechanism by which EBP50 interacted with EGFR and inhibited EGFR-mediated signaling.

In cervical cancer samples, EGFR signaling was overactivated in about $33 \%$ of CC patients $(30,38)$. Activated EGFR predicted poor response to (chemo)radiation and survival in CC, EGFR pathway was a promising therapeutic target for CC patients (30). The mechanisms for EGFR signaling activation were diverse, including egfr activating mutations $(39,40), e g f r$ gene amplification $(39,41-45)$ and disorder of EGFR signaling regulation $(4,18)$. In $32.63 \%$ of $\mathrm{CC}$ patients egfr gene activating mutations were found (46). In addition, TCGA dataset showed that $\operatorname{ErbB}$ and $e g f r$ alterations (including mutation and amplification) were detected in $\sim 17.7$ and $4 \%$ of CC cases, respectively. EBP50 failed to predict the prognosis of all CC patients. However, after ruling out patients with continuous activation mutation/copy number alteration of $e g f r / E r b B$ gene and (chemo)radiation which affected patient prognosis, EBP50 showed the prognosis predictive effect for CC patients. This further revealed predictive role of low EBP50 expression level for CC patients with poor prognosis was dependent on its interaction with EGFR and its regulatory role on EGFR signaling pathway.

In conclusion, this study demonstrated that EBP50 expression negatively correlated with CC cell proliferation, cell cycle and EGFR signaling activation. EBP50 knockdown abolished the inhibition on EGF-induced ERK signaling activation. The overexpression of EBP50 mutant DD disrupted the interaction with EGFR attenuated its inhibition on EGFR-mediated signaling, revealing EBP50 regulated EGFR signaling via interaction with EGFR. Further evidence showed that EBP50 could predict the prognosis of $\mathrm{CC}$ patients after ruling out the patients with continuous activation mutation/copy number alteration of $e g f r / E r b B$ gene and (chemo)radiation affecting patient prognosis. Our findings provided further insights into the molecular pathogenesis of cervical cancer and EBP50 could be a novel, precise therapeutic target and prognostic marker for CC patients.

\section{Acknowledgements}

This study was supported by the National Natural Science Foundation of the People's Republic of China (no. 30900247, 81372739).

\section{References}

1. Zhang W, Jiang Y, Yu Q, Qiang S, Liang P, Gao Y, Zhao X, Liu W and Zhang J: EGFR promoter methylation, EGFR mutation, and HPV infection in Chinese cervical squamous cell carcinoma. Appl Immunohistochem Mol Morphol 23: 661-666, 2015.

2. Ji M, Fan D, Yuan L, Zhang Y, Dong W and Peng X: EBP50 inhibits pancreatic cancer cell growth and invasion by targeting the $\beta$-catenin/E-cadherin pathway. Exp Ther Med 10: 1311-1316, 2015. 
3. Zheng JF, Sun LC, Liu H, Huang Y, Li Y and He J: EBP50 exerts tumor suppressor activity by promoting cell apoptosis and retarding extracellular signal-regulated kinase activity. Amino Acids 38: 1261-1268, 2010.

4. Clapéron A, Guedj N, Mergey M, Vignjevic D, DesboisMouthon C, Boissan M, Saubaméa B, Paradis V, Housset C and Fouassier L: Loss of EBP50 stimulates EGFR activity to induce EMT phenotypic features in biliary cancer cells. Oncogene 31 : 1376-1388, 2012

5. Molina JR, Agarwal NK, Morales FC, Hayashi Y, Aldape KD, Cote $\mathrm{G}$ and Georgescu MM: PTEN, NHERF1 and PHLPP form a tumor suppressor network that is disabled in glioblastoma. Oncogene 31: 1264-1274, 2012

6. Peng XL, Ji MY, Yang ZR, Song J and Dong WG: Tumor suppressor function of ezrin-radixin-moesin-binding phosphoprotein-50 through $\beta$-catenin/E-cadherin pathway in human hepatocellular cancer. World J Gastroenterol 19: 1306-1313, 2013

7. Ma Q, Jiao Y, Hao Y, Yan S, Lyu N, Gao H, Li D, Liu Q, Zheng J and Song N: Targeting of NHERF1 through RNA interference inhibits the proliferation and migration of metastatic prostate cancer cells. Oncol Lett 11: 1149-1154, 2016.

8. Bellizzi A, Malfettone A, Cardone RA and Mangia A: NHERF1/ EBP50 in breast cancer: Clinical perspectives. Breast Care (Basel) 5: 86-90, 2010.

9. Song J, Bai J, Yang W, Gabrielson EW, Chan DW and Zhang Z: Expression and clinicopathological significance of oestrogenresponsive ezrin-radixin-moesin-binding phosphoprotein 50 in breast cancer. Histopathology 51: 40-53, 2007

10. Cardone RA, Bellizzi A, Busco G, Weinman EJ, Dell'Aquila ME, Casavola V, Azzariti A, Mangia A, Paradiso A and Reshkin SJ: The NHERF1 PDZ2 domain regulates PKA-RhoA-p38-mediated NHE1 activation and invasion in breast tumor cells. Mol Biol Cell 18: 1768-1780, 2007.

11. Shibata T, Chuma M, Kokubu A, Sakamoto M and Hirohashi S EBP50, a beta-catenin-associating protein, enhances Wnt signaling and is over-expressed in hepatocellular carcinoma. Hepatology 38: 178-186, 2003.

12. Kislin KL, McDonough WS, Eschbacher JM, Armstrong BA and Berens ME: NHERF-1: Modulator of glioblastoma cell migration and invasion. Neoplasia 11: 377-387, 2009.

13. Yan J, Zhang Y, Ren C, Shi W and Chen L: Involvement of nuclear protein $\mathrm{C} 23$ in activation of EGFR signaling in cervical cancer. Tumour Biol 37: 905-910, 2016

14. Shostak K, Zhang X, Hubert P, Göktuna SI, Jiang Z, Klevernic I,

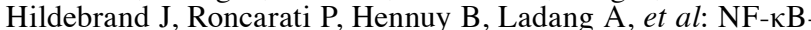
induced KIAA1199 promotes survival through EGFR signalling. Nat Commun 5: 5232, 2014.

15. Bhattacharyya RP, Reményi A, Yeh BJ and Lim WA: Domains, motifs, and scaffolds: The role of modular interactions in the evolution and wiring of cell signaling circuits. Annu Rev Biochem 75: 655-680, 2006.

16. Clapéron A and Therrien M: KSR and CNK: Two scaffolds regulating RAS-mediated RAF activation. Oncogene 26: 3143-3158, 2007.

17. Lazar CS, Cresson CM, Lauffenburger DA and Gill GN: The $\mathrm{Na}^{+} / \mathrm{H}^{+}$exchanger regulatory factor stabilizes epidermal growth factor receptors at the cell surface. Mol Biol Cell 15: 5470-5480, 2004

18. Yao W, Feng D, Bian W, Yang L, Li Y, Yang Z, Xiong Y, Zheng J, Zhai R and He J: EBP50 inhibits EGF-induced breast cancer cell proliferation by blocking EGFR phosphorylation. Amino Acids 43: 2027-2035, 2012.

19. Forni C, Braglia R, Lentini A, Nuccetelli M, Provenzano B, Tabolacci $\mathrm{C}$ and Beninati S: Role of transglutaminase 2 in quercetin-induced differentiation of B16-F10 murine melanoma cells. Amino Acids 36: 731-738, 2009.

20. Sun L, Zheng J, Wang Q, Song R, Liu H, Meng R, Tao T, Si Y, Jiang $\mathrm{W}$ and He J: NHERF1 regulates actin cytoskeleton organization through modulation of $\alpha$-actinin- 4 stability. FASEB J 30: 578-589, 2016

21. Yang L, Zheng J, Xiong Y, Meng R, Ma Q, Liu H, Shen H, Zheng S, Wang S and He J: Regulation of $\beta 2$-adrenergic receptor cell surface expression by interaction with cystic fibrosis transmembrane conductance regulator-associated ligand (CAL). Amino Acids 47: 1455-1464, 2015.

22. Wu SF, Qian WY, Zhang JW, Yang YB, Liu Y, Dong Y, Zhang ZB, Zhu YP and Feng YJ: Network motifs in the transcriptional regulation network of cervical carcinoma cells respond to EGF. Arch Gynecol Obstet 287: 771-777, 2013.
23. Herbst RS: Review of epidermal growth factor receptor biology. Int J Radiat Oncol Biol Phys 59 (Suppl): 21-26, 2004

24. Yang L, Wang Y, Chen $\mathrm{P}, \mathrm{Hu}$ J, Xiong Y, Feng D, Liu H, Zhang H, Yang $\mathrm{H}$ and $\mathrm{He} \mathrm{J}: \mathrm{Na}(+) / \mathrm{H}(+)$ exchanger regulatory factor 1 (NHERF1) is required for the estradiol-dependent increase of phosphatase and tensin homolog (PTEN) protein expression. Endocrinology 152: 4537-4549, 2011.

25. Maudsley S, Zamah AM, Rahman N, Blitzer JT, Luttrell LM, Lefkowitz RJ and Hall RA: Platelet-derived growth factor receptor association with $\mathrm{Na}(+) / \mathrm{H}(+)$ exchanger regulatory factor potentiates receptor activity. Mol Cell Biol 20: 8352-8363, 2000.

26. Pan Y, Weinman EJ and Dai JL: $\mathrm{Na}^{+} / \mathrm{H}^{+}$exchanger regulatory factor 1 inhibits platelet-derived growth factor signaling in breast cancer cells. Breast Cancer Res 10: R5, 2008

27. Wheeler DS, Barrick SR, Grubisha MJ, Brufsky AM, Friedman PA and Romero G: Direct interaction between NHERF1 and Frizzled regulates $\beta$-catenin signaling. Oncogene 30: 32-42, 2011.

28. Kreimann EL, Morales FC, de Orbeta-Cruz J, Takahashi Y, Adams H, Liu TJ, McCrea PD and Georgescu MM: Cortical stabilization of beta-catenin contributes to NHERF1/EBP50 tumor suppressor function. Oncogene 26: 5290-5299, 2007.

29. He J, Lau AG, Yaffe MB and Hall RA: Phosphorylation and cell cycle-dependent regulation of $\mathrm{Na}^{+} / \mathrm{H}^{+}$exchanger regulatory factor-1 by Cdc2 kinase. J Biol Chem 276: 41559-41565, 2001.

30. Noordhuis MG, Eijsink JJ, Ten Hoor KA, Roossink F, Hollema H, Arts HJ, Pras E, Maduro JH, Reyners AK, de Bock GH, et al: Expression of epidermal growth factor receptor (EGFR) and activated EGFR predict poor response to (chemo)radiation and survival in cervical cancer. Clin Cancer Res 15: 7389-7397, 2009.

31. Takahashi Y, Morales FC, Kreimann EL and Georgescu MM: PTEN tumor suppressor associates with NHERF proteins to attenuate PDGF receptor signaling. EMBO J 25: 910-920, 2006.

32. Kim HA, Kim KJ, Seo KH, Lee HK and Im SY: PTEN/MAPK pathways play a key role in platelet-activating factor-induced experimental pulmonary tumor metastasis. FEBS Lett 586: 4296-4302, 2012.

33. Shen H, Zhu F, Liu J, Xu T, Pei D, Wang R, Qian Y, Li Q, Wang L, Shi Z, et al: Alteration in Mir-21/PTEN expression modulates gefitinib resistance in non-small cell lung cancer. PLoS One 9: e103305, 2014.

34. Li QL, Gu FM, Wang Z, Jiang JH, Yao LQ, Tan CJ, Huang XY, Ke AW, Dai Z, Fan J, et al: Activation of PI3K/AKT and MAPK pathway through a PDGFR $\beta$-dependent feedback loop is involved in rapamycin resistance in hepatocellular carcinoma. PLoS One 7: e33379, 2012.

35. Zheng J, Dai Y, Yang Z, Yang L, Peng Z, Meng R, Xiong Y and He J: Ezrin-radixin-moesin-binding phosphoprotein-50 regulates EGF-induced AKT activation through interaction with EGFR and PTEN. Oncol Rep 35: 530-537, 2016.

36. Dard N and Peter M: Scaffold proteins in MAP kinase signaling: More than simple passive activating platforms. BioEssays 28: 146-156, 2006.

37. Cortese MS, Uversky VN and Dunker AK: Intrinsic disorder in scaffold proteins: Getting more from less. Prog Biophys Mol Biol 98: 85-106, 2008

38. Bumrungthai S, Munjal K, Nandekar S, Cooper K, Ekalaksananan T, Pientong $\mathrm{C}$ and Evans MF: Epidermal growth factor receptor pathway mutation and expression profiles in cervical squamous cell carcinoma: Therapeutic implications. J Transl Med 13: 244, 2015.

39. Giaccone G: Epidermal growth factor receptor inhibitors in the treatment of non-small-cell lung cancer. J Clin Oncol 23: 3235-3242, 2005.

40. Hynes NE and Lane HA: ERBB receptors and cancer: The complexity of targeted inhibitors. Nat Rev Cancer 5: 341-354, 2005.

41. Marquez A, Wu R, Zhao J, Tao J and Shi Z: Evaluation of epidermal growth factor receptor (EGFR) by chromogenic in situ hybridization (CISH) and immunohistochemistry (IHC) in archival gliomas using bright-field microscopy. Diagn Mol Pathol 13: 1-8, 2004.

42. Baselga $\mathbf{J}$ and Arteaga CL: Critical update and emerging trends in epidermal growth factor receptor targeting in cancer. J Clin Oncol 23: 2445-2459, 2005 
43. Takehana T, Kunitomo K, Suzuki S, Kono K, Fujii H, Matsumoto Y and Ooi A: Expression of epidermal growth factor receptor in gastric carcinomas. Clin Gastroenterol Hepatol 1: 438-445, 2003.

44. Al-Kuraya K, Schraml P, Torhorst J, Tapia C, Zaharieva B Novotny H, Spichtin H, Maurer R, Mirlacher M, Köchli O, et al: Prognostic relevance of gene amplifications and coamplifications in breast cancer. Cancer Res 64: 8534-8540, 2004.

45. Fallon KB, Palmer CA, Roth KA, Nabors LB, Wang W, Carpenter M, Banerjee R, Forsyth P, Rich K and Perry A: Prognostic value of 1p, 19q, 9p, 10q, and EGFR-FISH analyses in recurrent oligodendrogliomas. J Neuropathol Exp Neurol 63: 314-322, 2004
46. Qureshi R, Arora H, Biswas S, Perwez A, Naseem A, Wajid S, Gandhi G and Rizvi MA: Mutation analysis of EGFR and its correlation with the HPV in Indian cervical cancer patients. Tumour Biol: Jan 14, 2016 (Epub ahead of print). 